\title{
Uniform Boundedness and Global Existence of Solutions to a Quasilinear Diffusion Equation with Nonlocal Fisher-KPP Type Reaction Term
}

\author{
Xueyan Tao and Zhong Bo Fang*
}

\begin{abstract}
This paper deals with the Cauchy problem and Neumann initial boundary value problem for a quasilinear diffusion equation with nonlocal Fisher-KPP type reaction terms. We establish the uniform boundedness and global existence of solutions to the problems by using multipliers technique and modified Moser's iteration argument for some ranges of parameters. Moreover, the ranges of parameters have similar structure to that of the classical critical Fujita exponent.
\end{abstract}

\section{Introduction}

We investigate the following Cauchy problem $(\tau=0)$ and Neumann initial boundary value problem $(\tau=1)$ of the quasilinear diffusion equation with nonlocal Fisher-KPP type reaction term

$$
\begin{cases}u_{t}=\Delta u^{m}+u^{\alpha}\left(1-\int_{\Omega} u^{\beta} d x\right), & x \in \Omega, t>0, \\ \tau \frac{\partial u^{m}}{\partial \nu}=0, & x \in \partial \Omega, t>0 \\ u(x, 0)=u_{0}(x) \geq 0, & x \in \Omega,\end{cases}
$$

where $\tau \in\{0,1\}, m>0$, and $\alpha, \beta \geq 1$. When $\tau=0, \Omega$ is assumed to be $\mathbb{R}^{N}$, therefore (1.1) is a Cauchy problem; while in the case of $\tau=1$, we suppose that $\Omega \subset \mathbb{R}^{N}$ is a bounded domain with smooth boundary, hence (1.1) turns to a Neumann initial boundary value problem, $\nu$ is the outward unit normal vector on $\partial \Omega$. The nonnegative initial data $u_{0}$ is not identical to zero.

Nonlinear diffusion equations like (1.1) appear in various applications, which describes the diffusion of the concentration of Newtonian flow in a porous medium or the temperature of some combustible substances, see [6, 32]. In particular, equation (1.1) is a possible model for the diffusion system of some biological species, where $u(x, t)$ represents the density of the species at position $x$ and time $t, \Delta u^{m}$ portrays the mutation, which Received November 25, 2019; Accepted April 16, 2020.

Communicated by Cheng-Hsiung Hsu.

2010 Mathematics Subject Classification. 35A01, 35B33, 35K59.

Key words and phrases. quasilinear diffusion equation, nonlocal Fisher-KPP reaction, uniform boundedness, global existence.

*Corresponding author. 
we view as a spreading of the characteristic, and the reaction term $u^{\alpha}\left(1-\int_{\Omega} u^{\beta} d x\right)$ is considered as the rate of the reproduction. Due to the effect of spatial inhomogeneity, the occurrence of nonlocal term $\int_{\Omega} u^{\beta} d x$ denotes that the evolution of the species at a point in space depends not only on the density of species in partial region but also on the total region, refer to $2,14,18$. Moreover, as appeared in many literatures, e.g., 27, 28, 36], the nonlocal type reaction term $u^{\alpha}\left(1-\int_{\Omega} u^{\beta} d x\right)$ can also describe Darwinian evolution of a structured population density or the behaviors of cancer cells with therapy. In the nonlinear diffusion theory, there exist obvious differences among the situations of slow $(m>1)$, fast $(0<m<1)$, and linear $(m=1)$ diffusion. For example, there is a finite speed propagation in the slow and linear diffusion situations, whereas an infinite speed propagation exists in the case of fast diffusion.

During the past decades, there have been many works to deal with diffusion equations with local or nonlocal reaction terms, see [6, 22, 32, 34, 39]. Roughly, those works contain some focal topics such as existence and nonexistence of global solutions, blow-up phenomena, extinction phenomena, asymptotic behavior of the solutions as well as the critical exponent theory, etc. In this paper, we are particularly interested in the issues of global existence of solutions to the following quasilinear diffusion equation with nonlocal Fisher-KPP type reaction term

$$
u_{t}=\Delta u^{m}+F(t, u, I(u)), \quad x \in \Omega, t>0,
$$

where $I(u)=\int_{\Omega} u(y, t) d y$. To the best of our knowledge, compared with the local reaction problems, few results are available for such nonlocal models. When $m=1$ in 1.2 . Bebernes [5, 7], Pao [33] and Liu et al. [26] considered the thermal explosion model of compressible gas with the nonlocal reaction term

$$
F=e^{u}+a I\left(u_{t}\right) \quad \text { or } \quad F=e^{u}+a I\left(e^{u}\right)
$$

and they obtained the solvability, asymptotic properties and blow-up phenomena of solutions under null Dirichlet boundary condition. Wang et al. [37] investigated the semilinear diffusion model with the reaction term

$$
F=I\left(u^{\alpha}\right)-k u^{\gamma}, \quad \alpha, \gamma \geq 1
$$

and they derived sufficient condition for which the solutions exist globally and blow up in finite time by virtue of ODE analysis. Budd et al. [13], $\mathrm{Hu}$ and Yin [23] studied the partial differential equation with the following special nonlocal reaction term

$$
F=u^{\alpha}-\frac{1}{|\Omega|} I\left(u^{\alpha}\right)
$$

under null Neumann boundary condition, where $\alpha>1$. The solutions of these problems have a conservation property and, based on the convexity argument, they proved the 
nonexistence of global solutions under large initial energy. In addition, one can refer to [15 20,31 to review some latest researches on the nonlocal problems with fully nonlinear reaction terms, nonlocal semilinear parabolic equation with small positive initial energy and fourth-order thin-film equations. In [4], Anguiano et al. considered the diffusion equation with the reaction term

$$
F=f(u) I(u)(1-I(u))
$$

under null Dirichlet boundary condition, and they obtained the existence of global attractor. Recently, for the Cauchy problem and the Neumann initial boundary value problem with the nonlocal reaction term

$$
F=u^{\alpha}\left(1-I\left(u^{\beta}\right)\right)
$$

Bian and Chen studied the existence of global solutions and derived the critical exponents of the Fujita type (cf. [8,9]). Afterwards, Bian et al. [10] investigated the global existence and asymptotic behavior of solutions to Neumann initial boundary value problem with the nonlocal term

$$
F=u^{\alpha}(1-I(u))
$$

Besides, one can refer to the literature 12 to see studies on Fisher-KPP type equation with convolution operator.

When $m \neq 1$ in $[1.2$. Wang and Wo [38] investigated a fast diffusion equation with the nonlocal term

$$
F=u^{m}-I\left(u^{m}\right)
$$

under null Neumann boundary condition, and they proved a convergence of global solutions to some steady states in one-dimensional space. Fang et al. [16 studied the slow diffusion equation with the nonlocal term

$$
F=u^{\alpha} I\left(u^{\beta}\right)-u^{\gamma}
$$

under nonlocal boundary condition, and they established a new comparison principle and gave some sufficient conditions for which the solutions exist globally and blow up in finite time. Afterwards, $\mathrm{Xu}$ et al. [40] considered a fast diffusion equation with the nonlocal reaction term in [16] under a homogeneous Dirichlet boundary condition, and they derived some sufficient conditions for the extinction of nonnegative nontrivial weak solutions and the corresponding decay estimates by virtue of integral estimate method and ODE technique. In addition, for studies on the travelling fronts, entire solutions, and large-time behavior of solutions to local Fisher-KPP type diffusion problems, we refer to $21,24,25$ and the references therein. 
In view of the works mentioned above, much less effort has been devoted to the existence of uniformly bounded global solutions to quasilinear diffusion model (1.1). At a glance, our main difficulties lie in finding how the competitive relationship between the nonlinear diffusion term $\Delta u^{m}$ and the nonlinear nonlocal term $\int_{\Omega} u^{\beta} d x$ affect the global existence of solutions. In particular, the method used in the nonlocal semilinear diffusion problems in the aforementioned works of literatures (see [8, 9]) is no longer directly applicable to our nonlocal quasilinear model, therefore, we need more delicate analysis to obtain the global existence. Motivated by these observations, applying multipliers technique and modified Moser's iteration argument, we will show that the solutions to [1.1] are uniformly bounded and exist globally under appropriate conditions. Indeed, our results improve and generalize the Theorem 1 in [9] and [8], respectively.

Note that for $m>0$, the diffusion term $\Delta u^{m}(x, t)$ in problem (1.1) may be singular or degenerate on the set $\{(x, t) \mid u(x, t)=0\}$, which leads to the nonexistence of classical solutions to problem (1.1), therefore, we consider the weak solutions in the distribution sense. It is well-known that the existence of local weak solutions in time to problem (1.1) can be obtained by the fixed point theorems or standard parabolic regularity theory that can be applied to get suitable estimates in the standard limiting process (cf. [3, 35, 39]), and hence, we omit the details here. It can be seen that there exists $T_{\max }>0$ such that the unique weak solution $u$ to problem (1.1) satisfies

$$
u \in C\left(\left[0, T_{\max }\right) ; L^{\infty}(\Omega)\right),
$$

and either $T_{\max }=\infty$, or $T_{\max }$ is finite with $\lim \sup _{t \rightarrow T_{\max }}\|u(\cdot, t)\|_{L^{\infty}(\Omega)}=+\infty$, which is similar to the results in 29,30 .

Now we are ready to give our main results. For convenience, let $2^{*}$ denote the critical exponent of Sobolev embedding, that is,

$$
2^{*}= \begin{cases}\frac{2 N}{N-2} & \text { if } N \geq 3 \\ p \in(2, \infty) & \text { if } N=2 \\ \infty & \text { if } N=1\end{cases}
$$

We state the following existence of uniformly bounded global solutions in whole space $\mathbb{R}^{N}$ as well as in bounded domain, respectively.

Theorem 1.1. Let $\tau=0$. Suppose that $N \geq 1, m>\max \{0,1-2 / N\}, \beta \geq 1$ and that $\alpha$ satisfies the inequalities

$$
\max \{1, m-\beta\} \leq \alpha<m+\left(1-\frac{2}{2^{*}}\right) \beta .
$$

If the nonnegative initial data $u_{0}$ is in $L^{\beta}\left(\mathbb{R}^{N}\right) \cap L^{\infty}\left(\mathbb{R}^{N}\right)$, then the Cauchy problem (1.1) has a unique nonnegative global solution, which is uniformly bounded. 
Theorem 1.2. Let $\tau=1$. Suppose that $N \geq 1, m>0, \beta \geq 1$ and that $\alpha$ satisfies the inequalities

$$
\max \{1, m-\beta\} \leq \alpha<m+\frac{2}{N} \beta
$$

If the nonnegative initial data $u_{0}$ is in $L^{\infty}(\Omega)$, then the Neumann initial boundary value problem (1.1) has a unique nonnegative global solution, which is uniformly bounded.

Remark 1.3. From Theorems 1.1 and 1.2 we see that, for the existence of global solution to (1.1), Cauchy problem and Neumann initial boundary value problem share exactly the same critical exponent $\alpha_{c}=m+\frac{2}{N} \beta(N \geq 3)$. In fact, the structure of the critical exponent $\alpha_{c}$ is similar to that of the well-known classical critical Fujita exponent to the local porous medium equation with power like source term $u_{t}=\Delta u^{m}+u^{\alpha}$. As we all know, for the local problem, when $\alpha<\alpha_{c}$, the solutions blow up in finite time for any initial data (cf. 17, 19,29). However, Theorems 1.1 and 1.2 show the opposite result, i.e., global solution exists, which indicates that the nonlocal term has a huge influence on the properties of solutions.

The present work is organized as follows. In Section 2, we give a proof for Theorem 1.1 on global boundedness for the solution to the Cauchy problem $(\tau=0)$. In Section 3 , a proof for Theorem 1.2 on global boundedness for the solution to the Neumann initial boundary value problem $(\tau=1)$ is presented.

For simplicity, the variable of integral will be omitted without ambiguity, e.g., the integral $\int_{\Omega} f(x) d x$ is written as $\int_{\Omega} f(x)$.

\section{Global boundedness for $\tau=0$}

In this section, we consider global boundedness of the solution to the Cauchy problem 1.1. To begin with, we review Lemma 2 in $[9]$.

Lemma 2.1. [9] If $N \geq 1,1 \leq r<q<2^{*}$, and $\frac{q}{r}<\frac{2}{r}+1-\frac{2}{2^{*}}$, then for any $v \in H^{1}\left(\mathbb{R}^{N}\right) \cap L^{r}\left(\mathbb{R}^{N}\right)$, the following inequalities

$$
\begin{array}{rlrl}
\|v\|_{L^{q}\left(\mathbb{R}^{N}\right)}^{q} \leq & \varepsilon\|\nabla v\|_{L^{2}\left(\mathbb{R}^{N}\right)}^{2}+C \varepsilon^{-\frac{\lambda q}{2-\lambda q}}\|v\|_{L^{r}\left(\mathbb{R}^{N}\right)}^{\mu}, & & N \geq 3, \\
\|v\|_{L^{q}\left(\mathbb{R}^{N}\right)}^{q} \leq & \varepsilon_{1}\|\nabla v\|_{L^{2}\left(\mathbb{R}^{N}\right)}^{2}+\varepsilon_{2}\|v\|_{L^{2}\left(\mathbb{R}^{N}\right)}^{2} & \\
& +C\left(\varepsilon_{1}^{-\frac{\lambda q}{2-\lambda q}}+\varepsilon_{2}^{-\frac{\lambda q}{2-\lambda q}}\right)\|v\|_{L^{r}\left(\mathbb{R}^{N}\right)}^{\mu}, & N=1,2
\end{array}
$$

hold, where $C=C(N, q, r)$ and $\varepsilon, \varepsilon_{1}, \varepsilon_{2}>0$ are arbitrary constants, and

$$
\lambda=\frac{1 / r-1 / q}{1 / 2-1 / 2^{*}} \in(0,1), \quad \mu=\frac{2(1-\lambda) q}{2-\lambda q} .
$$

We now introduce the following key proposition to prove Theorem 1.1 . 
Proposition 2.2. Suppose that $N \geq 1, m>0, \beta \geq 1$ and that $\alpha$ satisfies the inequalities

$$
\max \{1, m-\beta\} \leq \alpha<m+\left(1-\frac{2}{2^{*}}\right) \beta .
$$

For any $T \in\left(0, T_{\max }\right)$ and each $\beta \leq k<\infty$, if the nonnegative initial data $u_{0}$ is in $\bigcap_{\beta \leq k<\infty} L^{k}\left(\mathbb{R}^{N}\right)$, then any solution to the Cauchy problem (1.1) satisfies

$$
\|u(\cdot, t)\|_{L^{k}\left(\mathbb{R}^{N}\right)} \leq C \quad \text { for all } t \in(0, T),
$$

where $C=C\left(N, m, \alpha, \beta, k,\left\|u_{0}\right\|_{L^{k}\left(\mathbb{R}^{N}\right)}\right)$.

Remark 2.3. Proposition 2.2 is a generalization of Proposition 2 in [9].

Proof of Proposition 2.2. We only give a proof for the case $N \geq 3$, since the cases of $N=1,2$ can be similarly proved. Multiplying the first equation in $(1.1)$ by $k u^{k-1}(k>1)$ and integrating the result over $\mathbb{R}^{N}$, we obtain the equation

$$
\frac{d}{d t} \int_{\mathbb{R}^{N}} u^{k}+\frac{4 m k(k-1)}{(k+m-1)^{2}} \int_{\mathbb{R}^{N}}\left|\nabla u^{\frac{k+m-1}{2}}\right|^{2}+k \int_{\mathbb{R}^{N}} u^{k+\alpha-1} \int_{\mathbb{R}^{N}} u^{\beta}=k \int_{\mathbb{R}^{N}} u^{k+\alpha-1}
$$

For each $k>\max \{1, \beta+1-\alpha\}$, we choose $k^{\prime}$ such that $k^{\prime}>\beta$ and $\max \left\{1, \frac{k+m-1}{2}\right\} \leq k^{\prime}<$ $k+\alpha-1$. Moreover, taking $v=u^{\frac{k+m-1}{2}}, q=\frac{2(k+\alpha-1)}{k+m-1}, r=\frac{2 k^{\prime}}{k+m-1}$, and $\varepsilon=\frac{2 m(k-1)}{(k+m-1)^{2}}$ in (2.1), we have

$$
\begin{aligned}
& \int_{\mathbb{R}^{N}} u^{k+\alpha-1}=\left\|u^{\frac{k+m-1}{2}}\right\|_{L^{q}\left(\mathbb{R}^{N}\right)}^{q} \\
\leq & \frac{2 m(k-1)}{(k+m-1)^{2}} \int_{\mathbb{R}^{N}}\left|\nabla u^{\frac{k+m-1}{2}}\right|^{2}+C_{1}\left(\frac{2 m(k-1)}{(k+m-1)^{2}}\right)^{-\frac{\lambda}{2-\lambda q}}\|u\|_{L^{k^{\prime}\left(\mathbb{R}^{N}\right)}}^{a},
\end{aligned}
$$

where $C_{1}=C_{1}\left(N, m, \alpha, k, k^{\prime}\right), \lambda=\frac{\frac{1}{k^{\prime}}-\frac{1}{k+\alpha-1}}{\frac{1}{k^{\prime}}-\frac{2}{2^{*}(k+m-1)}} \in(0,1), a=\frac{(1-\lambda)(k+\alpha-1)}{1-\frac{\lambda(k+\alpha-1)}{k+m-1}}$. Substituting (2.3) into 2.2 , there exists $C_{2}=k C_{1}\left(\frac{2 m(k-1)}{(k+m-1)^{2}}\right)^{-\frac{\lambda q}{2-\lambda q}}$ such that

$$
\frac{d}{d t} \int_{\mathbb{R}^{N}} u^{k}+\frac{2 m k(k-1)}{(k+m-1)^{2}} \int_{\mathbb{R}^{N}}\left|\nabla u^{\frac{k+m-1}{2}}\right|^{2}+k \int_{\mathbb{R}^{N}} u^{k+\alpha-1} \int_{\mathbb{R}^{N}} u^{\beta} \leq C_{2}\|u\|_{L^{k^{\prime}}\left(\mathbb{R}^{N}\right)^{\prime}}^{a}
$$

Since $\beta<k^{\prime}<k+\alpha-1$, the interpolation inequality yields that

$$
\begin{aligned}
\|u\|_{L^{k^{\prime}\left(\mathbb{R}^{N}\right)}}^{a} & \leq\|u\|_{L^{k+\alpha-1}\left(\mathbb{R}^{N}\right)}^{a \theta}\|u\|_{L^{\beta}\left(\mathbb{R}^{N}\right)}^{a(1-\theta)} \\
& =\left(\|u\|_{L^{k+\alpha-1}\left(\mathbb{R}^{N}\right)}^{k+\alpha-1}\|u\|_{L^{\beta}\left(\mathbb{R}^{N}\right)}^{\beta}\right)^{\frac{a \theta}{k+\alpha-1}}\|u\|_{L^{\beta}\left(\mathbb{R}^{N}\right)}^{a(1-\theta)-\frac{\beta a \theta}{k+\alpha-1}},
\end{aligned}
$$

where $\theta=\frac{\frac{1}{\beta}-\frac{1}{k^{\prime}}}{\frac{1}{\beta}-\frac{1}{k+\alpha-1}} \in(0,1)$. In addition, one can easily see the following equivalence

$$
\frac{a \theta}{k+\alpha-1}<1 \quad \Longleftrightarrow \quad \alpha<m+\frac{2}{N} \beta .
$$


Due to $\alpha+\beta \geq m$, we can choose $k^{\prime}=\frac{k+\alpha+\beta-1}{2}$ in 2.5 and get $a(1-\theta)-\frac{\beta a \theta}{k+\alpha-1}=0$. Applying Young's inequality to (2.5), we arrive at

$$
\begin{aligned}
C_{2}\|u\|_{L^{k^{\prime}\left(\mathbb{R}^{N}\right)}}^{a} & =C_{2}\left(\|u\|_{L^{k+\alpha-1}\left(\mathbb{R}^{N}\right)}^{k+\alpha-1}\|u\|_{L^{\beta}\left(\mathbb{R}^{N}\right)}^{\beta}\right)^{\frac{a \theta}{k+\alpha-1}} \\
& \leq \frac{k}{2} \int_{\mathbb{R}^{N}} u^{k+\alpha-1} \int_{\mathbb{R}^{N}} u^{\beta}+C_{3},
\end{aligned}
$$

where $C_{3}=\frac{k+\alpha-1-a \theta}{k+\alpha-1}\left(\frac{2 a \theta}{k(k+\alpha-1)}\right)^{\frac{a \theta}{k+\alpha-1-a \theta}} C_{2}^{\frac{k+\alpha-1}{k+\alpha-1-a \theta}}$. Substituting 2.6 into 2.4), we obtain the inequality

$$
\frac{d}{d t} \int_{\mathbb{R}^{N}} u^{k}+\frac{2 m k(k-1)}{(k+m-1)^{2}} \int_{\mathbb{R}^{N}}\left|\nabla u^{\frac{k+m-1}{2}}\right|^{2}+\frac{k}{2} \int_{\mathbb{R}^{N}} u^{k+\alpha-1} \int_{\mathbb{R}^{N}} u^{\beta} \leq C_{3}
$$

for each $k>\max \{1, \beta+1-\alpha\}$. It follows from Hölder's and Young's inequalities that

$$
\int_{\mathbb{R}^{N}} u^{\frac{k+\alpha+\beta-1}{2}} \leq \frac{k}{2} \int_{\mathbb{R}^{N}} u^{k+\alpha-1} \int_{\mathbb{R}^{N}} u^{\beta}+\frac{1}{2 k}
$$

In particular, picking $k=\alpha+\beta-1$ in (2.7) and (2.8) entails

$$
\frac{d}{d t} \int_{\mathbb{R}^{N}} u^{\alpha+\beta-1}+\int_{\mathbb{R}^{N}} u^{\alpha+\beta-1} \leq C_{3}+\frac{1}{2(\alpha+\beta-1)} .
$$

Applying a differential inequality technique to (2.9), we obtain

$$
\int_{\mathbb{R}^{N}} u^{\alpha+\beta-1} \leq \max \left\{\int_{\mathbb{R}^{N}} u_{0}^{\alpha+\beta-1}, C_{3}+\frac{1}{2(\alpha+\beta-1)}\right\} .
$$

Now, taking $k=\beta$ in $(2.2)$, we get the differential inequality

$$
\frac{d}{d t} \int_{\mathbb{R}^{N}} u^{\beta} \leq \beta \int_{\mathbb{R}^{N}} u^{\alpha+\beta-1}\left(1-\int_{\mathbb{R}^{N}} u^{\beta}\right)
$$

which results in $\int_{\mathbb{R}^{N}} u^{\beta} \leq \max \left\{\int_{\mathbb{R}^{N}} u_{0}^{\beta}, 1\right\}$. By the interpolation inequality, one can easily see that for all $k$ such that $\beta \leq k \leq \alpha+\beta-1$, the norm $\|u(\cdot, t)\|_{L^{k}\left(\mathbb{R}^{N}\right)}$ is uniform-in-time bounded.

When $\alpha+\beta-1<k<\infty$, taking $v=u^{\frac{k+m-1}{2}}, q=\widetilde{q}:=\frac{2 k}{k+m-1}, r=\frac{k+\alpha+\beta-1}{k+m-1}$ and $\varepsilon=\frac{m k(k-1)}{(k+m-1)^{2}}$ in 2.1 , and combining with 2.8 , we know that

$$
\begin{aligned}
& \int_{\mathbb{R}^{N}} u^{k}=\left\|u^{\frac{k+m-1}{2}}\right\|_{L^{\widetilde{q}}\left(\mathbb{R}^{N}\right)}^{\widetilde{q}} \\
\leq & \frac{m(k-1)}{(k+m-1)^{2}} \int_{\mathbb{R}^{N}}\left|\nabla u^{\frac{k+m-1}{2}}\right|^{2}+C_{4}\left(\frac{m k(k-1)}{(k+m-1)^{2}}\right)^{-\frac{\tilde{\lambda} \widetilde{q}}{2-\tilde{\lambda} \widetilde{q}}}\|u\|_{L^{\frac{k+\alpha+\beta-1}{2}}\left(\mathbb{R}^{N}\right)}^{\widetilde{a}} \\
\leq & \frac{m(k-1)}{(k+m-1)^{2}} \int_{\mathbb{R}^{N}}\left|\nabla u^{\frac{k+m-1}{2}}\right|^{2} \\
& +C_{4}\left(\frac{m k(k-1)}{(k+m-1)^{2}}\right)^{-\frac{\tilde{\lambda} \widetilde{q}}{2-\tilde{\lambda} \widetilde{q}}}\left(\int_{\mathbb{R}^{N}} u^{k+\alpha-1} \int_{\mathbb{R}^{N}} u^{\beta}\right)^{\frac{\widetilde{a}}{k+\alpha+\beta-1}},
\end{aligned}
$$


where $C_{4}=C_{4}(N, m, \alpha, \beta, k), \widetilde{\lambda}=\frac{\frac{1}{k+\alpha+\beta-1}-\frac{1}{2 k}}{\frac{1}{k+\alpha+\beta-1}-\frac{1}{2^{*}(k+m-1)}} \in(0,1), \widetilde{a}=\frac{k(1-\tilde{\lambda})}{1-\frac{k \tilde{\lambda}}{k+m-1}}$. It is easy to see that $\frac{\widetilde{a}}{k+\alpha+\beta-1}<1$. We apply Young's inequality to 2.10 to see

$$
\int_{\mathbb{R}^{N}} u^{k} \leq \frac{m(k-1)}{(k+m-1)^{2}} \int_{\mathbb{R}^{N}}\left|\nabla u^{\frac{k+m-1}{2}}\right|^{2}+\frac{k}{2} \int_{\mathbb{R}^{N}} u^{k+\alpha-1} \int_{\mathbb{R}^{N}} u^{\beta}+C_{5},
$$

where $C_{5}=C_{5}(N, m, \alpha, \beta, k)$. It follows from (2.7) and 2.11) that

$$
\frac{d}{d t} \int_{\mathbb{R}^{N}} u^{k}+\int_{\mathbb{R}^{N}} u^{k}+\frac{m k(k-1)}{(k+m-1)^{2}} \int_{\mathbb{R}^{N}}\left|\nabla u^{\frac{k+m-1}{2}}\right|^{2} \leq C_{3}+C_{5} .
$$

Therefore, for all $k$ such that $k>\alpha+\beta-1$, the norm $\|u(\cdot, t)\|_{L^{k}\left(\mathbb{R}^{N}\right)}$ is also uniform-in-time bounded.

The proof is complete.

Now we are ready to prove Theorem 1.1 .

Proof of Theorem 1.1. We also only need to prove the case $N \geq 3$. Let $q_{k}=2^{k}+\alpha+\beta-1$, where $k \in \mathbb{N}$. It is easy to see that $q_{k} \geq \beta$, and hence, the solution $u(\cdot, t)$ of the Cauchy problem is in $L^{q_{k}}\left(\mathbb{R}^{N}\right)$ for all $t \in(0, T)$ by Proposition 2.2. Taking $k=q_{k}$ in (2.2), we obtain the differential equation

$$
\begin{aligned}
& \frac{d}{d t} \int_{\mathbb{R}^{N}} u^{q_{k}}+\frac{4 m q_{k}\left(q_{k}-1\right)}{\left(q_{k}+m-1\right)^{2}} \int_{\mathbb{R}^{N}}\left|\nabla u^{\frac{q_{k}+m-1}{2}}\right|^{2}+q_{k} \int_{\mathbb{R}^{N}} u^{q_{k}+\alpha-1} \int_{\mathbb{R}^{N}} u^{q_{k}+\beta} \\
= & q_{k} \int_{\mathbb{R}^{N}} u^{q_{k}+\alpha-1} .
\end{aligned}
$$

The definition of $q_{k}$ enables us to pick $C_{m}:=4 m \min \left\{\frac{2}{(1+m)^{2}}, 1\right\}$ such that $\frac{4 m q_{k}\left(q_{k}-1\right)}{\left(q_{k}+m-1\right)^{2}} \geq$ $C_{m}>0$. Then it follows from 2.12 that

$$
\frac{d}{d t} \int_{\mathbb{R}^{N}} u^{q_{k}}+C_{m} \int_{\mathbb{R}^{N}}\left|\nabla u^{\frac{q_{k}+m-1}{2}}\right|^{2}+q_{k} \int_{\mathbb{R}^{N}} u^{q_{k}+\alpha-1} \int_{\mathbb{R}^{N}} u^{\beta} \leq q_{k} \int_{\mathbb{R}^{N}} u^{q_{k}+\alpha-1} .
$$

Taking $v=u^{\frac{q_{k}+m-1}{2}}, q=\bar{q}:=\frac{2\left(q_{k}+\alpha-1\right)}{q_{k}+m-1}, r=\frac{2 q_{k-1}}{q_{k}+m-1}$, and $\varepsilon=\frac{C_{m}}{2 q_{k}}$ in 2.1), we have

$$
\begin{aligned}
& \int_{\mathbb{R}^{N}} u^{q_{k}+\alpha-1}=\left\|u^{\frac{q_{k}+m-1}{2}}\right\|_{L^{\bar{q}}\left(\mathbb{R}^{N}\right)}^{\bar{q}} \\
\leq & \frac{C_{m}}{2 q_{k}} \int_{\mathbb{R}^{N}}\left|\nabla u^{\frac{q_{k}+m-1}{2}}\right|^{2}+C_{6}\left(\frac{C_{m}}{2 q_{k}}\right)^{-\frac{\bar{\lambda} \bar{q}}{2-\bar{\lambda} \bar{q}}}\left(\int_{\mathbb{R}^{N}} u^{q_{k-1}}\right)^{a_{1}},
\end{aligned}
$$

where $C_{6}=C_{6}(N, m, \alpha, \beta, k), \bar{\lambda}=\frac{\frac{1}{q_{k-1}}-\frac{1}{q_{k}+\alpha-1}}{\frac{1}{q_{k-1}}-\frac{2}{2^{*}\left(q_{k}+m-1\right)}} \in(0,1)$, and $a_{1}=1+\frac{q_{k}+\alpha-1-q_{k-1}}{q_{k-1}-\frac{N}{2}(\alpha-m)}<2$. Let

$$
\delta=\frac{\bar{\lambda} \bar{q}}{2}=\frac{q_{k}-q_{k-1}+\alpha-1}{q_{k}+m-1-\frac{2}{2^{*}} q_{k-1}}=g\left(2^{k-1}\right)
$$


where $g(y)=\frac{y+\alpha-1}{\left(1+\frac{2}{N}\right) y+\frac{2}{N}(\alpha+\beta-1)+m-1}$. It follows from $\alpha<m+\frac{2}{N} \beta$ that $g$ is increasing on $[1,+\infty)$, and hence, $0<g(1) \leq \delta \leq \frac{N}{N+2}<1$. From 2.14), we arrive at

$$
q_{k} \int_{\mathbb{R}^{N}} u^{q_{k}+\alpha-1} \leq \frac{C_{m}}{2} \int_{\mathbb{R}^{N}}\left|\nabla u^{\frac{q_{k}+m-1}{2}}\right|^{2}+C_{7} q_{k}^{\frac{\delta}{1-\delta}}\left(\int_{\mathbb{R}^{N}} u^{q_{k-1}}\right)^{a_{1}}
$$

where $C_{7}=C_{7}(N, m, \alpha, \beta)$. Similarly, taking $v=u^{\frac{q_{k}+m-1}{2}}, q=\frac{2 q_{k}}{q_{k}+m-1}, r=\frac{2 q_{k-1}}{q_{k}+m-1}$, and $\varepsilon=\frac{C_{m}}{2}$ in 2.1 , it can be obtained that

$$
\int_{\mathbb{R}^{N}} u^{q_{k}} \leq \frac{C_{m}}{2} \int_{\mathbb{R}^{N}}\left|\nabla u^{\frac{q_{k}+m-1}{2}}\right|^{2}+C_{8}\left(\int_{\mathbb{R}^{N}} u^{q_{k-1}}\right)^{a_{2}},
$$

where $C_{8}=C_{8}(N, m, \alpha, \beta)$ and $a_{2}=1+\frac{q_{k}-q_{k-1}}{q_{k-1}-\frac{N}{2}(m-1)}<2$. Adding 2.15 and 2.16 together, substituting the result into 2.13 , we can find a constant $C_{9}=\max \left\{C_{7}, C_{8}\right\}$ such that

$$
\begin{aligned}
\frac{d}{d t} \int_{\mathbb{R}^{N}} u^{q_{k}}+\int_{\mathbb{R}^{N}} u^{q_{k}} & \leq C_{7} q_{k}^{\frac{1}{1-\delta}}\left(\int_{\mathbb{R}^{N}} u^{q_{k-1}}\right)^{a_{1}}+C_{8}\left(\int_{\mathbb{R}^{N}} u^{q^{k-1}}\right)^{a_{2}} \\
& \leq C_{9}\left(q_{k}^{\frac{1}{1-\delta}}\left(\int_{\mathbb{R}^{N}} u^{q_{k-1}}\right)^{a_{1}}+\left(\int_{\mathbb{R}^{N}} u^{q_{k-1}}\right)^{a_{2}}\right) \\
& \leq 2 C_{9} q_{k}^{\frac{1}{1-\delta}} \max \left\{1,\left(\int_{\mathbb{R}^{N}} u^{q_{k-1}}\right)^{2}\right\} .
\end{aligned}
$$

By virtue of (2.17) and the well-known Moser-Alikakos iteration procedure (cf. [1], 11, Lemma 4.1] or Appendix), we derive that

$$
\|u(\cdot, t)\|_{L^{\infty}\left(\mathbb{R}^{N}\right)} \leq C \quad \text { for all } t \in(0, T),
$$

where $C=C\left(N, m, \alpha, \beta,\left\|u_{0}\right\|_{L^{\beta}\left(\mathbb{R}^{N}\right)},\left\|u_{0}\right\|_{L^{\infty}\left(\mathbb{R}^{N}\right)}\right)$. Since $T \in\left(0, T_{\max }\right)$ is arbitrary, 2.18) yields that $T_{\max }=\infty$, which completes the proof.

\section{Global boundedness for $\tau=1$}

In this section, we consider global boundedness of the solution to the Neumann initial boundary value problem (1.1). To begin with, we review two useful inequalities below.

Lemma 3.1. If $N \geq 1, p \geq 1, \gamma \in(0, p)$ and $w \in H^{1}(\Omega)$, then the Gagliardo-Nirenberg inequality

$$
\|w\|_{L^{p}(\Omega)} \leq c_{G N}\|w\|_{H^{1}(\Omega)}^{\sigma}\|w\|_{L^{\gamma}(\Omega)}^{1-\sigma}
$$

holds, where $c_{G N}=c_{G N}(N, p, \gamma), p(N-2)<2 N$, and $\sigma=\frac{1 / \gamma-1 / p}{1 / N-1 / 2+1 / \gamma} \in(0,1)$. In addition, if $s>0$, then the Poincaré inequality

$$
\|w\|_{H^{1}(\Omega)} \leq c_{P}\left(\|\nabla w\|_{L^{2}(\Omega)}+\|w\|_{L^{s}(\Omega)}\right)
$$

holds, where $c_{P}=c_{P}(N, s)$. 
We now introduce the following key proposition to prove Theorem 1.2 .

Proposition 3.2. Suppose that $N \geq 1, m>0, \beta \geq 1$ and that $\alpha$ satisfies the inequalities

$$
\max \{1, m-\beta\} \leq \alpha<m+\frac{2}{N} \beta .
$$

For any $T \in\left(0, T_{\max }\right)$ and each $k$ such that $1 \leq k<\infty$, if the nonnegative initial data $u_{0}$ is in $\bigcap_{\beta \leq k<\infty} L^{k}(\Omega)$, then any nonnegative solution to the Neumann initial boundary value problem (1.1) satisfies the inequality

$$
\|u(\cdot, t)\|_{L^{k}(\Omega)} \leq c \quad \text { for all } t \in(0, T),
$$

where $c=c\left(N, m, \alpha, \beta,\left\|u_{0}\right\|_{L^{k}(\Omega)}\right)$.

Remark 3.3. Proposition 3.2 is a generalization of Proposition 3 in [8].

Proof of Proposition 3.2. Multiplying the first equation in 1.1 by $k u^{k-1}(k>1)$ and integrating the result over $\Omega$, we have

$$
\frac{d}{d t} \int_{\Omega} u^{k}+\frac{4 m k(k-1)}{(k+m-1)^{2}} \int_{\Omega}\left|\nabla u^{\frac{k+m-1}{2}}\right|^{2}+k \int_{\Omega} u^{k+\alpha-1} \int_{\Omega} u^{\beta}=k \int_{\Omega} u^{k+\alpha-1} .
$$

For each $k>\max \{1, \beta+1-\alpha\}$, taking $w=u^{\frac{k+m-1}{2}}$ and $p=\frac{2(k+\alpha-1)}{k+m-1}$ in (3.1), and using (3.2), it can be obtained that

$$
\begin{aligned}
& k \int_{\Omega} u^{k+\alpha-1} \\
\leq & k c_{G N}^{p}\left(c_{P}\left(\left\|\nabla u^{\frac{k+m-1}{2}}\right\|_{L^{2}(\Omega)}^{2}+\left\|u^{\frac{k+m-1}{2}}\right\|_{L^{s}(\Omega)}^{2}\right)\right)^{\frac{p \sigma}{2}}\left\|u^{\frac{k+m-1}{2}}\right\|_{L^{\gamma}(\Omega)}^{p(1-\sigma)} \\
\leq & c_{1}\left\|\nabla u^{\frac{k+m-1}{2}}\right\|_{L^{2}(\Omega)}^{2 \frac{p \sigma}{2}}\left\|u^{\frac{k+m-1}{2}}\right\|_{L^{\gamma}(\Omega)}^{\frac{2 p(1-\sigma)}{2} \frac{2-p \sigma}{2}}+c_{1}\left\|u^{\frac{k+m-1}{2}}\right\|_{L^{s}(\Omega)}^{p \sigma}\left\|u^{\frac{k+m-1}{2}}\right\|_{L^{\gamma}(\Omega)}^{p(1-\sigma)} \\
\leq & \frac{2 m k(k-1)}{(k+m-1)^{2}} \int_{\Omega}\left|\nabla u^{\frac{k+m-1}{2}}\right|^{2}+c_{2}\left\|u^{\frac{k+m-1}{2}}\right\|_{L^{\gamma}(\Omega)}^{\frac{2 p(1-\sigma)}{2}} \\
& +c_{1}\left\|u^{\frac{k+m-1}{2}}\right\|_{L^{s}(\Omega)}^{p \sigma}\left\|u^{\frac{k+m-1}{2}}\right\|_{L^{\gamma}(\Omega)}^{p(1-\sigma)},
\end{aligned}
$$

where $\left(\frac{N(p-2)}{2}\right)_{+}<\gamma<p, s>0, r=\frac{2 k^{\prime}}{k+m-1}, \sigma=\frac{1 / \gamma-1 / p}{1 / N-1 / 2+1 / \gamma} \in(0,1)$, and $c_{1}, c_{2}$ depend on $N, m, \alpha, \gamma$ and $k$. Substituting (3.4) into (3.3), we get

$$
\begin{aligned}
& \frac{d}{d t} \int_{\Omega} u^{k}+\frac{2 m k(k-1)}{(k+m-1)^{2}} \int_{\Omega}\left|\nabla u^{\frac{k+m-1}{2}}\right|^{2}+k \int_{\Omega} u^{k+\alpha-1} \int_{\Omega} u^{\beta} \\
\leq & c_{2}\left\|u^{\frac{k+m-1}{2}}\right\|_{L^{\gamma}(\Omega)}^{\frac{2 p(1-\sigma)}{2-p \sigma}}+c_{1}\left\|u^{\frac{k+m-1}{2}}\right\|_{L^{s}(\Omega)}^{p \sigma}\left\|u^{\frac{k+m-1}{2}}\right\|_{L^{\gamma}(\Omega)}^{p(1-\sigma)} .
\end{aligned}
$$

With $\gamma=\frac{k+\alpha+\beta-1}{k+m-1}$, Hölder's inequality yields

$$
\left\|u^{\frac{k+m-1}{2}}\right\|_{L^{\gamma}(\Omega)} \leq\left(\int_{\Omega} u^{k+\alpha-1} \int_{\Omega} u^{\beta}\right)^{\frac{k+m-1}{2(k+\alpha+\beta-1)}} .
$$


In addition, it can be shown that the following equivalence

$$
\frac{k+m-1}{k+\alpha+\beta-1} \cdot \frac{p(1-\sigma)}{2-p \sigma}<1 \quad \Longleftrightarrow \quad \alpha<m+\frac{2}{N} \beta
$$

holds, which yields that

$$
\begin{aligned}
c_{2}\left\|u^{\frac{k+m-1}{2}}\right\|_{L^{\gamma}(\Omega)}^{\frac{2 p(1-\sigma)}{2-p \sigma}} & \leq c_{2}\left(\int_{\Omega} u^{k+\alpha-1} \int_{\Omega} u^{\beta}\right)^{\frac{k+m-1}{k+\alpha+\beta-1} \cdot \frac{p(1-\sigma)}{2-p \sigma}} \\
& \leq \frac{k}{4} \int_{\Omega} u^{k+\alpha-1} \int_{\Omega} u^{\beta}+c_{3},
\end{aligned}
$$

and

$$
\begin{aligned}
& c_{1}\left\|u^{\frac{k+m-1}{2}}\right\|_{L^{s}(\Omega)}^{p \sigma}\left\|u^{\frac{k+m-1}{2}}\right\|_{L^{\gamma}(\Omega)}^{p(1-\sigma)} \\
\leq & c_{1}\left\|u^{\frac{k+m-1}{2}}\right\|_{L^{s}(\Omega)}^{p \sigma}\left(\int_{\Omega} u^{k+\alpha-1} \int_{\Omega} u^{\beta}\right)^{\frac{p(k+m-1)(1-\sigma)}{2(k+\alpha+\beta-1)}} \\
\leq & \frac{k}{4} \int_{\Omega} u^{k+\alpha-1} \int_{\Omega} u^{\beta}+c_{4}\left\|u^{\frac{k+m-1}{2}}\right\|_{L^{s}(\Omega)}^{\chi},
\end{aligned}
$$

where $\chi=\frac{2 p \sigma(k+\alpha+\beta-1)}{2(k+\alpha+\beta-1)-p(k+m-1)(1-\sigma)}>0$, and $c_{3}, c_{4}$ depend on $N, m, \alpha, \beta$ and $k$. Substituting (3.6) and (3.7) into (3.5), we get the inequality

$$
\begin{aligned}
& \frac{d}{d t} \int_{\Omega} u^{k}+\frac{2 m k(k-1)}{(k+m-1)^{2}} \int_{\Omega}\left|\nabla u^{\frac{k+m-1}{2}}\right|^{2}+\frac{k}{2} \int_{\Omega} u^{k+\alpha-1} \int_{\Omega} u^{\beta} \\
\leq & c_{3}+c_{4}\left\|u^{\frac{k+m-1}{2}}\right\|_{L^{s}(\Omega)}^{\chi} .
\end{aligned}
$$

We utilize Hölder's inequality $\left(\int_{\Omega} u^{\beta}\right)^{\frac{k+\alpha-1}{\beta}} \leq|\Omega|^{\frac{k+\alpha-1-\beta}{\beta}} \int_{\Omega} u^{k+\alpha-1}$ to estimate

$$
\left(\int_{\Omega} u^{\beta}\right)^{\frac{k+\alpha+\beta-1}{\beta}} \leq|\Omega|^{\frac{k+\alpha-1-\beta}{\beta}} \int_{\Omega} u^{k+\alpha-1} \int_{\Omega} u^{\beta} .
$$

Taking $s=\frac{2 \beta}{k+m-1}$, and therefore,

$$
\frac{\beta \chi}{s(k+\alpha+\beta-1)}=\frac{p \sigma(k+m-1)}{2(k+\alpha+\beta-1)-p(k+m-1)(1-\sigma)}<1 .
$$

It follows from 3.9 that

$$
\begin{aligned}
c_{4}\left\|u^{\frac{k+m-1}{2}}\right\|_{L^{s}(\Omega)}^{\chi} & =c_{4}\left(\int_{\Omega} u^{\beta}\right)^{\frac{k+\alpha+\beta-1}{\beta} \cdot \frac{\beta \chi}{s(k+\alpha+\beta-1)}} \\
& \leq \frac{k}{4}|\Omega|^{\frac{\beta+1-\alpha-k}{\beta}}\left(\int_{\Omega} u^{\beta}\right)^{\frac{k+\alpha+\beta-1}{\beta}}+c_{5} \\
& \leq \frac{k}{4} \int_{\Omega} u^{k+\alpha-1} \int_{\Omega} u^{\beta}+c_{5},
\end{aligned}
$$


where $c_{5}=c_{5}(N, m, \alpha, \beta, k)$. Substituting (3.10) into (3.8), we have

$$
\frac{d}{d t} \int_{\Omega} u^{k}+\frac{2 m k(k-1)}{(k+m-1)^{2}} \int_{\Omega}\left|\nabla u^{\frac{k+m-1}{2}}\right|^{2}+\frac{k}{4} \int_{\Omega} u^{k+\alpha-1} \int_{\Omega} u^{\beta} \leq c_{3}+c_{5} .
$$

In particular, taking $k=\beta$ in (3.11), from $(3.9)$ we can choose $M_{1}$ and $M_{2}$ depending on $N, m, \alpha$ and $\beta$ such that

$$
\frac{d}{d t} \int_{\Omega} u^{\beta}+M_{1}\left(\int_{\Omega} u^{\beta}\right)^{\frac{\alpha+2 \beta-1}{\beta}} \leq M_{2} .
$$

Differential inequality $(3.12)$ results in

$$
\int_{\Omega} u^{\beta} \leq \max \left\{\int_{\Omega} u_{0}^{\beta},\left(\frac{M_{2}}{M_{1}}\right)^{\frac{\beta}{\alpha+2 \beta-1}}\right\} .
$$

Therefore, we obtain the uniform boundedness for $\|u(\cdot, t)\|_{L^{\beta}(\Omega)}$. Thanks to Hölder's inequality, it can be seen that

$$
\|u(\cdot, t)\|_{L^{k}(\Omega)} \leq c\left(k, N, m, \alpha, \beta,\left\|u_{0}\right\|_{L^{\beta}(\Omega)}\right)
$$

for all $k$ such that $1 \leq k<\beta$ and $t \in(0, T)$.

For any $k>\max \left\{\beta, m-1, \frac{N}{2}(1-m)\right\}$, taking $w=u^{\frac{k+m-1}{2}}, p=p_{1}:=\frac{2 k}{k+m-1}$ in (3.1), we know that the inequality

$$
\int_{\Omega} u^{k}=\left\|u^{\frac{k+m-1}{2}}\right\|_{L^{p_{1}(\Omega)}}^{p_{1}} \leq c_{6}\left\|u^{\frac{k+m-1}{2}}\right\|_{H^{1}(\Omega)}^{p_{1} \sigma_{1}}\left\|u^{\frac{k+m-1}{2}}\right\|_{L^{\gamma_{1}}(\Omega)}^{p_{1}\left(1-\sigma_{1}\right)}
$$

holds for each $\gamma_{1} \in\left(0, p_{1}\right)$, where $c_{6}=c_{6}\left(N, m, \gamma_{1}, k\right)$ and $\sigma_{1}=\frac{1 / \gamma_{1}-1 / p_{1}}{1 / N-1 / 2+1 / \gamma_{1}} \in(0,1)$. Taking $\gamma_{1}=\frac{2 \beta}{k+m-1}$, it is easy to see that $p_{1} \sigma_{1}<2$. It then follows from the boundedness of $\|u(\cdot, t)\|_{L^{\beta}(\Omega)}, 3.2$ and 3.13 that

$$
\begin{aligned}
\int_{\Omega} u^{k} & \leq c_{7}\left\|u^{\frac{k+m-1}{2}}\right\|_{H^{1}(\Omega)}^{p_{1} \sigma_{1}} \\
& \leq c_{8}\left\|\nabla u^{\frac{k+m-1}{2}}\right\|_{L^{2}(\Omega)}^{2 \cdot \frac{p_{1} \sigma_{1}}{2}}+c_{8}\left\|u^{\frac{k+m-1}{2}}\right\|_{L^{\gamma_{1}}(\Omega)}^{p_{1} \sigma_{1}} \\
& \leq \frac{m k(k-1)}{(k+m-1)^{2}} \int_{\Omega}\left|\nabla u^{\frac{k+m-1}{2}}\right|^{2}+c_{9},
\end{aligned}
$$

where $c_{7}, c_{8}, c_{9}$ depend on $k, N, m, \alpha, \beta$, and $\left\|u_{0}\right\|_{L^{\beta}(\Omega)}$. Substituting 3.14 into (3.11, we obtain the differential inequality

$$
\frac{d}{d t} \int_{\Omega} u^{k}+\int_{\Omega} u^{k} \leq c_{10}
$$

where $c_{10}=c_{10}\left(k, N, m, \alpha, \beta,\left\|u_{0}\right\|_{L^{\beta}(\Omega)}\right)$, and hence, by an argument of differential inequality, 3.15 results in

$$
\int_{\Omega} u^{k} \leq \max \left\{\int_{\Omega} u_{0}^{\beta}, c_{10}\right\}
$$


for any $k>\max \left\{\beta, m-1, \frac{N}{2}(1-m)\right\}$.

When $\beta \leq k<\max \left\{\beta, m-1, \frac{N}{2}(1-m)\right\}$, we apply the interpolation inequality to derive the uniform boundedness for $\|u(\cdot, t)\|_{L^{k}(\Omega)}$ and, from which, we can find a constant $c=c\left(k, N, m, \alpha, \beta,\left\|u_{0}\right\|_{L^{k}(\Omega)}\right)$ such that

$$
\|u(\cdot, t)\|_{L^{k}(\Omega)} \leq c \quad \text { for all } k \geq 1 \text { and each } t \in(0, T)
$$

The proof is completed.

Now we are ready to prove Theorem 1.2 .

Proof of Theorem 1.2. Using a similar argument as the one used in the proof of Theorem 1.1 and applying the Moser-Alikakos technique [1, it can be shown that the solution $u(\cdot, t)$ to problem $(1.1)$ satisfies

$$
\|u(\cdot, t)\|_{L^{\infty}(\Omega)} \leq c \quad \text { for all } t \in(0, T)
$$

where $c=c\left(N, m, \alpha, \beta,\left\|u_{0}\right\|_{L^{\infty}(\Omega)}\right)$. Since $T \in\left(0, T_{\max }\right)$ is arbitrary, (3.16) yields that $T_{\max }=\infty$, which completes the proof.

\section{A. Appendix}

For completeness, we give a detailed proof for (2.18).

Proof of 2.18. Let $\rho=\frac{1}{1-\delta}=O(1)$. Then $\rho>1$, thanks to 2.17), we can find a constant $C=C(N, m, \alpha, \beta)>1$ such that

$$
\frac{d}{d t} \int_{\mathbb{R}^{N}} u^{q_{k}}+\int_{\mathbb{R}^{N}} u^{q_{k}} \leq C q_{k}^{\rho} \max \left\{1,\left(\int_{\mathbb{R}^{N}} u^{q_{k-1}}\right)^{2}\right\} .
$$

Setting $y_{k}(t)=\int_{\mathbb{R}^{N}} u^{q_{k}}$, we have from A.1 that

$$
\begin{aligned}
y_{k}(t) & \leq y_{k}(0) e^{-t}+C q_{k}^{\rho} \max \left\{1, \sup _{t \geq 0} y_{k-1}^{2}(t)\right\}\left(1-e^{-t}\right) \\
& \leq y_{k}(0)+C q_{k}^{\rho} \max \left\{1, \sup _{t \geq 0} y_{k-1}^{2}(t)\right\}
\end{aligned}
$$

Let $M:=\max \left\{1,\left\|u_{0}\right\|_{L^{\infty}\left(\mathbb{R}^{N}\right)},\left\|u_{0}\right\|_{L^{\beta}\left(\mathbb{R}^{N}\right)}\right\}$. The interpolation inequality yields that

$$
y_{k}(0)=\int_{\mathbb{R}^{N}} u_{0}^{q_{k}} \leq \frac{\beta}{q_{k}}\left\|u_{0}\right\|_{L^{\beta}\left(\mathbb{R}^{N}\right)}^{q_{k}}+\frac{q_{k}-\beta}{q_{k}}\left\|u_{0}\right\|_{L^{\infty}\left(\mathbb{R}^{N}\right)}^{q_{k}} \leq M^{q_{k}}
$$


Substituting A.3 into A.2 , there exist $\bar{C}, \widetilde{C}$ depend on $N, \alpha, \beta$ and $M$, such that

$$
\begin{aligned}
y_{k}(t) & \leq M^{q_{k}}+C q_{k}^{\rho} \max \left\{1, \sup _{t \geq 1} y_{k-1}^{2}(t)\right\} \\
& \leq 2 C q_{k}^{\rho} \max \left\{M^{q_{k}}, \sup _{t \geq 0} y_{k-1}^{2}(t)\right\} \\
& \leq \bar{C} 2^{\rho k} \max \left\{M^{q_{k}}, \sup _{t \geq 0} y_{k-2}^{2^{2}}(t)\right\} \\
& \leq \widetilde{C} \bar{C}^{1+2} 2^{\rho(k+2(k-1))} \max \left\{M^{q_{k}}, \sup _{t \geq 0} y_{k-2}^{2^{2}}(t)\right\} \\
& \leq \cdots \\
& \leq \widetilde{C} \bar{C}^{\sum_{j=0}^{k-1} 2^{j}} 2^{\rho \sum_{j=0}^{k-1} 2^{j}(k-j+1)} \max \left\{M^{q_{k}}, \sup _{t \geq 0} y_{0}^{2^{k}}(t)\right\} \\
& =\widetilde{C} \bar{C}^{2^{k-1}} 2^{\rho\left(2^{k+1}-k\right)} \max \left\{M^{q_{k}}, \sup _{t \geq 0} y_{0}^{2^{k}}(t)\right\},
\end{aligned}
$$

here we have used the fact $q_{k}=2^{k}+\alpha+\beta-1 \leq(\alpha+\beta) 2^{k}$. Therefore,

$$
\int_{\mathbb{R}^{N}} u^{q_{k}}=y_{k}^{\frac{1}{q_{k}}} \leq C(N, \alpha, \beta, M) .
$$

Letting $k \rightarrow \infty$, we obtain the desired result (2.18).

\section{Acknowledgments}

The second author is supported by the Natural Science Foundation of Shandong Province of China (Grant No. ZR2019MA072) and the Fundamental Research Funds for the Central Universities (No. 201964008). The authors would like to deeply thank all the reviewers for their insightful and constructive comments.

\section{References}

[1] N. D. Alikakos, $L^{p}$ bounds of solutions of reaction-diffusion equations, Comm. Partial Differential Equations 4 (1979), no. 8, 827-868.

[2] W. Allegretto, G. Fragnelli, P. Nistri and D. Papini, Coexistence and optimal control problems for a degenerate predator-prey model, J. Math. Anal. Appl. 378 (2011), no. $2,528-540$.

[3] J. R. Anderson, Local existence and uniqueness of solutions of degenerate parabolic equations, Comm. Partial Differential Equations 16 (1991), no. 1, 105-143. 
[4] M. Anguiano, P. E. Kloeden and T. Lorenz, Asymptotic behaviour of nonlocal reaction-diffusion equations, Nonlinear Anal. 73 (2010), no. 9, 3044-3057.

[5] J. Bebernes and A. Bressan, Thermal behavior for a confined reactive gas, J. Differential Equations 44 (1982), no. 1, 118-133.

[6] J. Bebernes and D. Eberly, Mathematical Problems from Combustion Theory, Applied Mathematical Sciences 83, Springer-Verlag, New York, 1989.

[7] J. Bebernes and R. Ely, Comparison techniques and the method of lines for a parabolic functional equation, Rocky Mountain J. Math. 12 (1982), no. 4, 723-733.

[8] S. Bian, Global solutions to a nonlocal Fisher-KPP type problem, Acta Appl. Math. 147 (2017), 187-195.

[9] S. Bian and L. Chen, A nonlocal reaction diffusion equation and its relation with Fujita exponent, J. Math. Anal. Appl. 444 (2016), no. 2, 1479-1489.

[10] S. Bian, L. Chen and E. A. Latos, Global existence and asymptotic behavior of solutions to a nonlocal Fisher-KPP type problem, Nonlinear Anal. 149 (2017), 165-176.

[11] S. Bian, J.-G. Liu and C. Zou, Ultra-contractivity for Keller-Segel model with diffusion exponent $m>1-2 / d$, Kinet. Relat. Models 7 (2014), no. 1, 9-28.

[12] N. F. Britton, Aggregation and the competitive exclusion principle, J. Theoret. Biol. 136 (1989), no. 1, 57-66.

[13] C. Budd, B. Dold and A. Stuart, Blowup in a partial differential equation with conserved first integral, SIAM J. Appl. Math. 53 (1993), no. 3, 718-742.

[14] À. Calsina, C. Perelló and J. Saldaña, Non-local reaction-diffusion equations modelling predator-prey coevolution, Publ. Mat. 38 (1994), no. 2, 315-325.

[15] Z. Dong and J. Zhou, Global existence and finite time blow-up for a class of thin-film equation, Z. Angew. Math. Phys. 68 (2017), no. 4, Paper No. 89, 17 pp.

[16] Z. B. Fang, J. Zhang and S.-C. Yi, Roles of weight functions to a nonlocal porous medium equation with inner absorption and nonlocal boundary condition, Abstr. Appl. Anal. 2012 (2012), Art. ID 326527, 16 pp.

[17] H. Fujita, On the blowing up of solutions of the Cauchy problem for $u_{t}=\Delta u+u^{1+\alpha}$, J. Fac. Sci. Univ. Tokyo Sect. I 13 (1966), 109-124.

[18] J. Furter and M. Grinfeld, Local vs. nonlocal interactions in population dynamics, J. Math. Biol. 27 (1989), no. 1, 65-80. 
[19] V. A. Galaktionov, S. P. Kurdyumov, A. P. Mikhailov and A. A. Samarskii, Unbounded solutions of the Cauchy problem for the parabolic equation $u t=\nabla(u \sigma \nabla u)+$ $u \beta$, Soviet Phys. Dokl. 25 (1980), 458-459.

[20] W. Gao and Y. Han, Blow-up of a nonlocal semilinear parabolic equation with positive initial energy, Appl. Math. Lett. 24 (2011), no. 5, 784-788.

[21] F. Hamel and N. Nadirashvili, Travelling fronts and entire solutions of the FisherKPP equation in $\mathbb{R}^{N}$, Arch. Ration. Mech. Anal. 157 (2001), no. 2, 91-163.

[22] B. Hu, Blow-up Theories for Semilinear Parabolic Equations, Lecture Notes in Mathematics 2018, Springer, Heidelberg, 2011.

[23] B. Hu and H.-M. Yin, Semilinear parabolic equations with prescribed energy, Rend. Circ. Mat. Palermo (2) 44 (1995), no. 3, 479-505.

[24] J. R. King and P. M. McCabe, On the Fisher-KPP equation with fast nonlinear diffusion, R. Soc. Lond. Proc. Ser. A Math. Phys. Eng. Sci. 459 (2003), no. 2038, $2529-2546$.

[25] W.-T. Li, Y.-J. Sun and Z.-C. Wang, Entire solutions in the Fisher-KPP equation with nonlocal dispersal, Nonlinear Anal. Real World Appl. 11 (2010), no. 4, 2302-2313.

[26] Q. Liu, Y. Chen and S. Lu, Uniform blow-up profiles for nonlinear and nonlocal reaction-diffusion equations, Nonlinear Anal. 71 (2009), no. 5-6, 1572-1583.

[27] A. Lorz, T. Lorenzi, J. Clairambault, A. Escargueil and B. Perthame, Modeling the effects of space structure and combination therapies on phenotypic heterogeneity and drug resistance in solid tumors, Bull. Math. Biol. 77 (2015), no. 1, 1-22.

[28] A. Lorz, S. Mirrahimi and B. Perthame, Dirac mass dynamics in multidimensional nonlocal parabolic equations, Comm. Partial Differential Equations 36 (2011), no. 6, 1071-1098.

[29] K. Mochizuki and K. Mukai, Existence and nonexistence of global solutions to fast diffusions with source, Methods Appl. Anal. 2 (1995), no. 1, 92-102.

[30] K. Mukai, K. Mochizuki and Q. Huang, Large time behavior and life span for a quasilinear parabolic equation with slowly decaying initial values, Nonlinear Anal. 39 (2000), no. 1, Ser. A: Theory Methods, 33-45.

[31] C. P. Niculescu and I. Rovenţa, Large solutions for semilinear parabolic equations involving some special classes of nonlinearities, Discrete Dyn. Nat. Soc. 2010 (2010), Art. ID 491023, 11 pp. 
[32] C. V. Pao, Nonlinear Parabolic and Elliptic Equations, Plenum Press, New York, 1992.

[33] _ Blowing-up of solution for a nonlocal reaction-diffusion problem in combustion theory, J. Math. Anal. Appl. 166 (1992), no. 2, 591-600.

[34] P. Quittner and P. Souplet, Superlinear Parabolic Problems: Blow-up, Global Existence and Steady States, Birkhäuser Verlag, Basel, 2007.

[35] P. E. Sacks, Continuity of solutions of a singular parabolic equation, Nonlinear Anal. 7 (1983), no. 4, 387-409.

[36] V. Volpert and V. Vougalter, Existence of stationary pulses for nonlocal reactiondiffusion equations, Doc. Math. 19 (2014), 1141-1153.

[37] M. Wang and Y. Wang, Properties of positive solutions for non-local reaction-diffusion problems, Math. Methods Appl. Sci. 19 (1996), no. 14, 1141-1156.

[38] X. Wang and W. Wo, Long time behavior of solutions for a scalar nonlocal reactiondiffusion equation, Arch. Math. (Basel) 96 (2011), no. 5, 483-490.

[39] Z. Wu, J. Zhao, J. Yin and H. Li, Nonlinear Diffusion Equations, World Scientific, River Edge, NJ, 2001.

[40] X. Xu, Z. B. Fang and S.-C. Yi, Extinction and decay estimates of solutions for a porous medium equation with nonlocal source and strong absorption, Bound. Value Probl. 2013 (2013), Article number 24, 13 pp.

Xueyan Tao

School of Mathematical Sciences, Peking University, Beijing 100871, China

E-mail address: taoxueyankitty@163.com

Zhong Bo Fang

School of Mathematical Sciences, Ocean University of China, Qingdao 266100, China

E-mail address: fangzb7777@hotmail.com 